\title{
Evaluation of non-English dietary supplement advertisements in an ethnic minority community in America
}

\author{
Eunice P Chung ${ }^{1, *}$, Hye J Hwang ${ }^{1}$ and Myo-Kyoung Kim ${ }^{2}$ \\ 'Western University of Health Sciences, College of Pharmacy, Pomona, CA 91766-1854, USA: \\ ${ }^{2}$ University of the Pacific, School of Pharmacy, Stockton, CA, USA
}

Submitted 27 January 2006: Accepted 11 0ctober 2006: First published online 5 March 2007

\begin{abstract}
Objective: The primary objective was to evaluate the rate at which non-English dietary supplement advertisements distributed in a sampled ethnic minority community are in compliance with the federal advertising regulations. The secondary objective was to assess the availability of supporting evidence to substantiate the advertised health claims.

Design: Cross-sectional study.

Setting: The contents of dietary supplement advertisements from the Los Angeles Korea Times and the Los Angeles Korea Daily were evaluated during the month of July 2005. After removing duplicate advertisements, the percentage of advertisements making prohibited disease claims and DSHEA (Dietary Supplement Health and Education Act) disclaimer statements was determined. The presence of data substantiating advertised claims was determined by requesting data from the manufacturers and browsing the manufacturers' websites. An observational technique was utilised for content analysis, and data analysis was conducted using quantitative descriptive statistics.

Results: Disease claims were present in $84.5 \%$, while DHSEA disclaimer statements were present in only $18.4 \%$ of the advertisements. Data to substantiate the claims were provided by $53.4 \%$ of the manufacturers. The majority of the additional information consisted of repetition of the advertised claims and consumer testimonies. Experimental data were available for only $13.6 \%$ of the products.

Conclusions: The high rate of non-compliance with federal regulations suggests a need for better oversight of non-English promotions of dietary supplements.
\end{abstract}

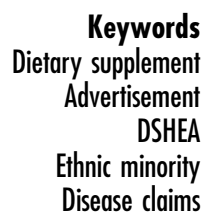

Sales of dietary supplements in the USA have increased dramatically since enactment of the Dietary Supplement Health and Education Act of 1994 (DSHEA) ${ }^{1}$. A survey showed that $52 \%$ of the US adult population uses at least one dietary supplement ${ }^{2}$. A dietary supplement is defined as any product taken by mouth that contains a 'dietary ingredient' intended to supplement the $\operatorname{diet}^{3}$. Therefore, dietary supplements may include vitamins, minerals, herbs or other botanicals, amino acids, and substances such as enzymes, organ tissues, glandular extracts and metabolites. Because DSHEA categorised dietary supplements as a subset of food, not as drugs, manufacturers are not required to provide evidence to substantiate the safety and efficacy, nor obtain approval by the Food and Drug Administration (FDA). Once the product is marketed, the burden is on the FDA to prove that a dietary supplement is harmful in order to take regulatory actions ${ }^{4}$.

Manufacturers of dietary supplements are allowed to make three types of claims: health claims (e.g. 'calcium may reduce risk of osteoporosis'); nutrient claims (e.g. 'high calcium'); and structure/function claims (e.g (promotes healthy joints') $)^{5}$. However, manufacturers are not permitted to make disease claims, which are defined as claims specific to treatment, prevention or cure of a specific disease or condition (e.g. 'treats osteoarthritis'). Such claims relating to diseases are limited to drugs. The FDA regulates the labelling of dietary supplements and works closely with the Federal Trade Commission (FTC), which has jurisdiction over the advertising of dietary supplements.

According to the FTC's advertising guide for dietary supplements, the advertising must be truthful, not misleading, and claims must be substantiated by adequate evidence $^{6}$. Enforcing such regulations, however, is challenging due to limited resources. With increasing reports of harmful effects caused by dietary supplements, there are growing concerns within the medical community regarding insufficient oversight of dietary supplements, resulting in increased public health risks ${ }^{7-9}$.

The dietary supplements advertised in languages other than English add another layer of complexity and therefore may pose a greater regulatory challenge to the FTC. 
However, insufficient control of dietary supplements advertised in languages other than English could potentially endanger the health of minorities in the USA. According to the Bureau of Labor Statistics and the Census Bureau, 13373000 Asian-Americans (16\% of the US population) do not have health insurance ${ }^{10}$. Since dietary supplements are readily accessible and make overwhelmingly promising health claims, important treatment choices can be made without consulting a physician, particularly for minorities without health insurance. To our knowledge, the compliance rate of the non-English dietary supplement advertisements circulated in ethnic minority communities to US regulatory standards has not been evaluated.

The first objective of this study was to evaluate the contents of dietary supplement advertisements in nonEnglish newspapers distributed in a sampled ethnic minority community to determine the rate of compliance with DSHEA and the FTC dietary supplement advertising guidelines. The second objective was to assess the availability of supporting evidence to substantiate the advertised health claims.

\section{Methods}

\section{Sample selection}

A Korean-American community within the metropolitan Los Angeles area, with over a half-million Korean-American residents, was selected as the sample ethnic minority community for our study. The two newspapers with the highest reported circulation within the Korean-American community were identified as the Los Angeles Korea Times (LAKT) and the Los Angeles Korea Daily (LAKD), circulating 80000 and 70000 copies per day, respectively. All dietary supplement advertisements in LAKT and LAKD during July 2005 were collected. Only the advertisements put out by the manufacturer or the distributor were included for analysis. The dietary supplements that appeared as part of a chain store advertisement were excluded. Duplicate advertisements were eliminated in order to represent each unique advertisement only once. The products in the advertisements were subcategorised into herbal/botanical, vitamins, mineral or amino acid to evaluate the type of dietary supplements being promoted in the selected minority community.

\section{Content analysis}

The contents of each advertisement were reviewed in detail to determine their compliance with the DSHEA and the FTC's dietary supplement advertising regulations. The analysis was conducted concurrently by two pharmacists familiar with the regulations and fluent in both English and Korean. The first measure for compliance with these guidelines was the usage of 'disease claims', which is permitted only for drugs. In order to differentiate 'disease claims' from 'health claims' and/or 'structure and function claims' that are permitted for dietary supplements, the definition and description provided by the Center for Food Safety and Applied Nutrition (CFSAN) of the FDA was adopted $^{4}$. The advertisements claiming to treat, prevent or cure diseases were categorised as containing 'disease claims'. According to the FTC advertising guideline, both expressed and implied disease claims are considered inappropriate. Therefore, the disease claims were further classified as being explicit or implicit. The disease claims were considered 'explicit' if specific names of the diseases were written in the advertisement, along with the words treat, prevent or cure. The implicit disease claims were determined based on the net impression conveyed by the entire advertisement, including the text, the name of the product and the depictions. In addition, disease states (i.e. diabetes or hypertension) used in the advertisements were categorised to determine the common diseases targeted by the dietary supplement advertisers.

The second measure to evaluate compliance of the dietary supplement was the use of the DSHEA disclaimers in advertising. Under DSHEA, the product labels should contain two disclaimers: 'the statement has not been evaluated by FDA' and 'the product is not intended to diagnose, treat, cure, or prevent any disease'. Although DHSEA does not mandate these disclaimers for dietary supplement advertisements, insertion of the disclaimers is recommended to prevent consumers from being misled or deceived $^{5}$. The advertisements with and without these disclaimers were counted.

\section{Evaluation of supporting evidence for claims}

In 2004, the FDA announced that dietary supplement claims must be substantiated by 'high quality evidence"12. In addition, the FTC's truth-in-advertising law mandates that advertisers must have adequate substantiation for all product claims before disseminating an advertisement ${ }^{5}$. In this study, documents to substantiate the advertised claims were requested by contacting the manufacturers by E-mail and/or by telephone. Supporting evidence provided by the manufacturers was evaluated in terms of study types such as in vitro studies, animal studies and/or human clinical trials. For advertisements that provided Internet sites for further information, the contents on the websites were also reviewed.

\section{Data analysis}

Descriptive statistics (\%) were adopted to describe the results of the study. The numbers of advertisements with compliant or non-compliant claims were counted and divided by the total number of unique dietary supplement advertisements.

\section{Results}

\section{Sample selection}

A total of 470 separate advertisements were collected from LAKT and LAKD during July 2005. After removing the 
duplicate advertisements within and across the two newspapers, 103 unique dietary supplement advertisements were identified. The majority of the advertisements listed 'herbals or botanicals' (56.3\%), followed by 'minerals' (7.8\%) and 'amino acids' (7.8\%) as active ingredients, whereas $35.9 \%$ of the dietary supplements did not list the ingredients.

\section{Content analysis}

Disease claims prohibited for dietary supplements were found in 87 of the 103 (84.5\%) advertisements. The advertised dietary supplements made up to 12 different disease claims per product, with a mean of $3.01 \pm 2.86$ diseases. All but one of the disease claims were determined to be explicit. Table 1 shows the list of disease states commonly targeted by the advertised dietary supplements. The targeted diseases were primarily common chronic illnesses and cancer.

Only $18.4 \%$ of the advertisements contained DSHEA warnings. Of note, the DSHEA warnings were present at a much lower rate among the advertisements with prohibited disease claims (14.9\%) compared with the advertisements that did not make any disease claims (37.5\%).

\section{Evaluation of supporting evidence for claims}

Only $31.3 \%$ of the manufacturers provided additional information when requested to provide evidence to substantiate the advertised claims. The majority of the additional information was repetition of the advertised claims or individual testimonies by previous consumers. Information on experimental data, consisting mostly of animal studies or small human studies, was provided for only $13.6 \%$ of the products.

\section{Discussion}

This study demonstrated that dietary supplement advertisements in the studied ethnic minority community have an alarmingly high rate of non-compliance with federal regulations, suggesting insufficient regulatory oversight for the non-English advertisements. While further comprehensive investigations should be conducted to

Table 1 Top seven diseases which the dietary supplement advertisements claimed to be able to treat, prevent or cure $(n=103)$

\begin{tabular}{lc}
\hline Diseases & Number of products $(\%)^{*}$ \\
\hline Diabetes & $34(33.0)$ \\
Cancer & $28(27.2)$ \\
Hypertension & $27(26.2)$ \\
Arthritis $\dagger$ & $20(19.4)$ \\
Hypercholesterolaemia & $17(16.5)$ \\
Sexual dysfunction & $17(16.5)$ \\
Obesity & $14(13.6)$ \\
\hline
\end{tabular}

* The sum of the percentages in the table exceeds $100 \%$ due to products making multiple disease claims.

† Rheumatoid or osteoarthritis. generalise the findings to all ethnic minority communities, we suspect that similar problems exist in other ethnic minority communities.

The DSHEA warnings were found in only a small fraction of the advertisements. Although it was not an objective of this study, it was observed that all the DSHEA warnings, when present, were written in English, while the rest of the advertisement was presented in Korean. Considering that the sampled newspapers were targeted at the non-English-speaking population, this implies an attempt to conceal the facts from consumers and should be investigated further in future studies. None of the supporting evidence provided could be considered as 'high quality' evidence, since it consisted mostly of consumer testimonials and details of animal experiments, with a small number of human trials insufficiently powered to validate the results.

The misleading advertisements can potentially jeopardise public health by influencing consumers to replace effective medications with the advertised dietary supplements. Consumers without medical training are vulnerable to miraculous and often 'too good to be true' claims. It has been shown that a majority of regular dietary supplement users believe the advertised claims made by the manufacturers ${ }^{11}$. Considering the presence of many ethnic minority communities throughout America and the large number of uninsured residents in these communities that rely on non-English media, the scale and the impact of the problem cannot be underestimated.

Our study is limited by the fact that the evaluation was conducted concurrently by the two investigators without assessing the inter-evaluator reproducibility. The other major limitation is that the study reflects problems in only one selected minority community. A larger study investigating dietary supplement advertisements in multiple minority communities concomitantly and comparing the results with the advertisements published in English newspapers would further validate the findings from this study.

\section{Conclusion}

The intent of DSHEA was to guarantee the availability of food products so that the consumers can freely make informed choices about their health ${ }^{12}$. However, our findings suggest that the non-English dietary supplement advertisements targeted at the ethnic minority community may be influencing consumer decisions by making claims often prohibited by the DSHEA or unsubstantiated by adequate research. Therefore, further comprehensive investigations on the non-English dietary supplement advertisements should be performed to unveil the prevalence and the impact of misleading advertisements among the minority populations in the USA. In addition, the high rate of non-compliance with government regulations suggests that better oversight of non-English promotions of 
dietary supplements is essential for the protection of the public health of minority groups in the USA.

\section{Acknowledgements}

Sources of funding: This was a non-funded study.

Conflict of interest declaration: The authors declare that there are no financial or other conflicts of interest related to the content of this study.

Authorship responsibilities: E.P.C. was responsible for the inception of the idea. Both E.P.C. and H.J.H. were involved in data collection. M.-K.K. assisted with analysis and interpretation of the data. All of the authors were involved in preparing and reviewing the manuscript.

Acknowledgements: The authors would like to thank Dr Rajul Patel for providing consultation on the manuscript.

\section{References}

1 Pinco RG, Halpern TH. Guidelines for the promotion of dietary supplements: examining government regulation five years after enactment of the Dietary Supplement Health and Education Act of 1994. Food and Drug Law Journal 1999; 54: 567-85.

2 Radimer K, Bindewald B, Hughes J, Ervin B, Swanson C, Picciano MF. Dietary supplement use by US adults: data from the National Health and Nutrition Examination Survey, 1999-2000. American Journal of Epidemiology 2004; 160 $339-49$.

3 US Food and Drug Administration. Overview of dietary supplements [online], 2001. Available from http://vm.cfsan. fda.gov/ dms/ds-oview.html. Accessed June 2005.
4 US Food and Drug Administration (Center for Food Safety and Applied Nutrition). Claims that can be made for conventional food and dietary supplements [online], September 2003. Available from http://www.cfsan.fda.gov/ $\sim$ dms/hclaims.html. Accessed July 2005.

5 Federal Trade Commission. Dietary supplements: an advertising guide for industry [online], April 2001. Available from http://www.ftc.gov/bcp/conline/pubs/buspubs/dietsupp.htm. Accessed July 2005.

6 Fontanarosa PB, Rennie D, DeAngelis CD. The need for regulation of dietary supplements - lessons from ephedra. Journal of the American Medical Association 2003; 289: 1568-70.

7 Marcus DM, Grollmand AP. Botanical medicines - the need for new regulations. New England Journal of Medicine 2002; 347: 2073-5.

8 Lewis JD, Strom BL. Balancing safety of dietary supplements with the free market. Annals of Internal Medicine 2002; 136: 616-8.

9 US Census Bureau. Health insurance coverage status and type of coverage by selected characteristics [online], 2004. Available from http://pubdb3.census.gov/macro/032005/ health/h01_000.htm. Accessed September 2005.

10 US Food and Drug Administration. FDA announces major initiatives for dietary supplements [online], 2004. Available from http://www.fda.gov/bbs/topics/news/2004/NEW01130.html. Accessed September 2005.

11 Blendon RJ, DesRoches CM, Benson JM, Brodie M, Altman DE. Americans' views on the use and regulation of dietary supplements. Archives of Internal Medicine 2001; 161: 805-10.

12 Khatcheressain LA. Regulation of dietary supplements: five years of DSHEA. Food and Drug Law Journal 1999; 54: 623-44. 